\title{
PROOF OF A CONJECTURE OF HELSON ${ }^{1}$
}

\author{
BY WALTER RUDIN
}

\section{Communicated by H. Helson, March 1, 1968}

Let $m_{n}$ denote the Haar measure of the torus $T^{n}$, the distinguished boundary of the unit polydisc $U^{n}$ in the space of $n$ complex variables. If $f$ is holomorphic in $U^{n}$, define

$$
f^{*}(z)=\lim _{r \rightarrow 1} f(r z)
$$

for those $z \in T^{n}$ for which this radial limit exists. Here $z=\left(z_{1}, \cdots, z_{n}\right)$, $r z=\left(r z_{1}, \cdots, r z_{n}\right)$. The various $H^{p}$-norms in $U^{n}$, for $0<p<\infty$, $n=1,2,3, \cdots$, are defined by

$$
\|f\|_{p, n}=\sup _{0<r<1}\left\{\int_{T^{n}}|f(r z)|^{p} d m_{n}(z)\right\}^{1 / p} .
$$

As in one variable, the inequality

$$
\log |f(0)| \leqq \int_{T^{n}} \log \left|f^{*}(z)\right| d m_{n}(z)
$$

holds for every $f \in H^{p}\left(U^{n}\right)$. Define

$$
\Delta(f)=\int_{T^{n}} \log \left|f^{*}(z)\right| d m_{n}(z)-\log |f(0)| .
$$

For $f \in H^{2}\left(U^{n}\right)$, let $S(f)$ denote the $H^{2}$-closure of the set of all products $P f$, where $P$ ranges over the polynomials in $n$ variables; $S(f)$ is the invariant subspace of $H^{2}\left(U^{n}\right)$ generated by $f$.

A very well-known theorem of Beurling states (in one variable) that

$$
S(f)=H^{2}(U) \text { if and only if } \Delta(f)=0 .
$$

One of these implications holds equally well for several variables, as has been known for quite some time to Helson and Lowdenslager: If $f \in H^{2}\left(U^{n}\right)$ and $S(f)=H^{2}\left(U^{n}\right)$, then $\Delta(f)=0$. Here is a sketch of a simple proof: (i) $\Delta(P f)=\Delta(P)+\Delta(f) \geqq \Delta(f)$ for all $P$. (ii) $\Delta$ is an upper semicontinuous function on $H^{2}\left(U^{n}\right)$. (iii) Therefore $\Delta(g) \geqq \Delta(f)$ for every $g \in S(f)$.

1 Research partially supported by NSF Grant GP-6764. 
Helson has conjectured [1, p. 23] that the converse is false for $n=2$ (hence also for $n>2$ ). (Actually, Helson stated the problem somewhat differently, in terms that involve only the boundary values of the functions under consideration.) This conjecture is correct:

Theorem. There exists a function $f \in H^{2}\left(U^{2}\right)$ such that $\Delta(f)=0$ but $S(f) \neq H^{2}\left(U^{2}\right)$.

The proof depends on the following two observations.

(I) If $F \in H^{\infty}(U)$, if $F$ has no zero in $U$, and if $f \in H^{\infty}\left(U^{2}\right)$ is defined by

$$
f\left(z_{1}, z_{2}\right)=F\left(\left(z_{1}+z_{2}\right) / 2\right),
$$

then $\Delta(f)=0$.

(II) Associate to each $f \in H^{2}\left(U^{2}\right)$ the function

$$
(\Psi f)(\lambda)=f((1+\lambda) / 2(1+\lambda) / 2) \quad(\lambda \in U) .
$$

If $0<p<\frac{1}{2}$, there is a constant $C_{p}<\infty$ such that

$$
\|\Psi f\|_{p, 1} \leqq C_{p}\|f\|_{2,2} \text {. }
$$

Thus $\Psi$ maps $H^{2}\left(U^{2}\right)$ into $H^{p}(U)$ if $p<\frac{1}{2}$. Note that $\Psi f$ is essentially the restriction of $f$ to a certain disc in $U^{2}$ which touches $T^{2}$ at just one point.

ProOF OF (I). If $|\alpha|=1, z \rightarrow \alpha z$ is a measure-preserving map of $T^{2}$ onto $T^{2}$. Hence

$$
\int_{T^{2}} d m_{2}(z) \int_{T} \log \left|f^{*}(\alpha z)\right| d m_{1}(\alpha)=\int_{T^{2}} \log \left|f^{*}(z)\right| d m_{2}(z),
$$

as is seen by interchanging the integrations on the left.If $z=\left(z_{1}, z_{2}\right) \in T^{2}$, if $z_{1} \neq z_{2}$, and if $2 w=z_{1}+z_{2}$, then $|w|<1$, so that

$$
\log |F(0)|=\int_{T} \log |F(\alpha w)| d m_{1}(\alpha) .
$$

This says that the inner integral on the left of (9) is equal to $\log |f(0)|$ whenever $z_{1} \neq z_{2}$, which is true for almost all $z \in T^{2}$. Hence $\Delta(f)=0$.

PROOF OF (II). For simplicity, assume $\|f\|_{2,2}=1$. Apply the Schwarz inequality to the Cauchy formula

$$
f(\zeta, \zeta)=\int_{T^{2}} \frac{f^{*}\left(z_{1}, z_{2}\right)}{\left(1-\bar{z}_{1} \zeta\right)\left(1-\bar{z}_{2} \zeta\right)} d m_{2}(z)
$$

to obtain the estimate 


$$
\begin{aligned}
|f(\zeta, \zeta)| & \leqq\left\{\int_{T^{2}}\left|1-\bar{z}_{1} \zeta\right|^{-2}\left|1-\bar{z}_{2} \zeta\right|^{-2} d m_{2}(z)\right\}^{1 / 2} \\
& =\int_{T}|1-\bar{w} \zeta|^{-2} d m_{1}(w)=\left(1-|\zeta|^{2}\right)^{-1}
\end{aligned}
$$

if $|\zeta|<1$. For $\lambda=r e^{i \theta}, 0<r<1$, it follows that

$$
|(\Psi f)(\lambda)| \leqq\left\{1-|(1+\lambda) / 2|^{2}\right\}^{-1} \leqq\left\{r \sin ^{2}(\theta / 2)\right\}^{-1}
$$

which gives (8) with

$$
C_{p}=\left\{\frac{1}{2 \pi} \int_{-\pi}^{\pi}|\sin (\theta / 2)|^{-2 p} d \theta\right\}^{1 / p} .
$$

Proof of the Theorem. Put $F(\lambda)=\exp \{(\lambda+1) /(\lambda-1)\}$ and associate $f$ with $F$ as in (I). Then $\Delta(f)=0$.

Fix $p, 0<p<\frac{1}{2}$. If $P$ is any polynomial in two variables, (II) gives

$$
\|1-P f\|_{2,2} \geqq C_{p}^{-1}\|1-\Psi P \cdot \Psi f\|_{p, 1} .
$$

Note that $(\Psi f)(\lambda)=e^{-1} F^{2}(\lambda)$. Thus $e \Psi f$ is a nontrivial inner function in $U$. Since multiplication by an inner function is an isometry in $H^{p}(U)$ (relative to the metric given by $\|g-h\|_{p, 1}^{p}$ if $p<1$ ) one sees that $H^{p}(U) \Psi f$ is a closed subspace of $H^{p}(U)$ which does not contain 1 . The right side of (10) is therefore bounded below by some positive constant, and so (10) implies that 1 is not in $S(f)$. Hence $S(f) \neq H^{2}\left(U^{2}\right)$.

\section{REFERENCE}

1. Henry Helson, Lectures on invariant subspaces, Academic Press, New York, 1964. UNIVERSITY OF WisCONSIN 